\title{
Verificando la integridad del registro arqueológico: análisis de fábricas en las unidades arqueológicas del paleolítico medio/superior de la Cova Gran (Santa Linya, Lleida)
}

\author{
Miquel Roy Sunyer, ${ }^{1}$ Xavier Roda Gilabert, ${ }^{1}$ Alfonso Benito-Calvo, ${ }^{2}$ Jorge \\ Martínez-Moreno, ${ }^{1}$ Rafael Mora Torcal ${ }^{3}$
}

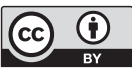

Recibido: 28-10-2014

Aceptado: 10-11-2014

\section{Resumen}

El análisis de fábricas, es decir, la orientación y el ángulo de buzamiento de los objetos, constituye una técnica resolutiva en el estudio de los procesos de formación de yacimientos. En este artículo presentamos una síntesis de las principales aproximaciones realizada alrededor de esta técnica de análisis aplicada en los últimos años en conjuntos arqueológicos, así como su aplicación en el caso de los niveles de la transición paleolítico medio/superior de la Cova Gran. Dicha metodología se integra en nuestro método de excavación, que hemos sistematizado a lo largo de los años con el desarrollo de modelos teóricos y aplicaciones tecnológicas (Mora, 1994; Martínez-Moreno et al., 2011). Aportando nuevos datos a estudios previos, se comparan las fábricas sedimentarias frente a las arqueológicas de dichos niveles, hallando diferencias significativas entre estos dos tipos de fábricas. Tales observaciones, junto con otros atributos, denotan procesos de formación diferenciados que señalan la incidencia de la actividad humana en los procesos tafonómicos de los artefactos arqueológicos así como una escasa incidencia de perturbaciones sin/postdeposicionales en este tramo de la secuencia de la Cova Gran.

Palabras clave: fábricas sedimentarias; fábricas arqueológicas; pisoteo; procesos de formación; Cova Gran.

1. Centre d'Estudis del Patrimoni Arqueològic de la Prehistòria (CEPAP-UAB). Universitat Autònoma de Barcelona. Facultat de Lletres. 08193 Bellaterra (Cerdanyola del Vallès).

miquel.roy@uab.cat; javier.roda@uab.cat; jorge.martinez@uab.cat

2. Centro Nacional de Investigación sobre la Evolución Humana (CENIEH). Paseo Sierra de Atapuerca s/n. 09002 Burgos.

alfonso.benito@cenieh.es

3. Centre d'Estudis del Patrimoni Arqueològic de la Prehistòria (CEPAP-UAB). Universitat Autònoma de Barcelona. Facultat de Lletres. 08193 Bellaterra (Cerdanyola del Vallès). Programa ICREA-Academia. Generalitat de Catalunya. España.

rafael.mora@uab.cat 
Miquel Roy Sunyer, Xavier Roda Gilabert, Alfonso Benito-Calvo, Jorge Martínez-Moreno, Rafael Mora Torcal
Verificando la integridad del registro arqueológico: análisis de fábricas en las unidades arqueológicas del paleolítico medio/superior de la Cova Gran (Santa Linya, Lleida)

Resum. Verificant la integritat del registre arqueològic: anàlisi de fabriques a les unitats arqueològiques del paleolític mitjà/superior de la Cova Gran (Santa Linya, Lleida)

L'anàlisi de fabriques, és a dir, l'orientació i l'angle de cabussament dels objectes, constitueix una tècnica resolutiva en l'estudi dels processos de formació de jaciments. En aquest article presentem una síntesi de les principals aproximacions realitzades al voltant d'aquesta tècnica d'anàlisi aplicada durant els últims anys en conjunts arqueològics, així com la seva aplicació en el cas dels nivells de la transició paleolític mitjà/superior de la Cova Gran. Tal metodologia s'integra en el nostre mètode d'excavació, que hem sistematitzat al llarg dels anys amb el desenvolupament de models teòrics i aplicacions tecnològiques (Mora, 1994; Martínez-Moreno et al., 2011). Aportant noves dades a estudis previs, es comparen les fabriques sedimentàries enfront de les arqueològiques dels nivells mencionats, i afloren diferències significatives entre aquests dos tipus de fabriques. Aquestes observacions, conjuntament amb altres atributs, denoten processos de formació diferenciats que assenyalen la incidència de l'activitat humana en els processos tafonòmics dels artefactes arqueològics, així com una escassa incidència de pertorbacions sin/postdeposicionals en aquest tram de la seqüència de la Cova Gran.

Paraules clau: fabriques sedimentàries; fabriques arqueològiques; trepig; processos de formació; Cova Gran.

\begin{abstract}
Assessing the integrity of the archaeological record: fabric analysis of the archaeological units in the Middle/Upper Paleolithic of Cova Gran (Santa Linya, Lleida)

Fabric analysis, that is the study of orientation and slope of the objects, constitutes a resolving technique in the study of site formation processes. In this paper we present a synthesis of the different approaches undertaken around this technique applied during the last years in archaeological contexts, and it application in the case of Middle/Upper Paleolithic levels of Cova Gran. This methodology is integrated in our excavation method that we have systematized along the last years with the development of theoretical models and technological applications (Mora 1994, Martínez-Moreno et al. 2011). We provide new data to previous studies through the comparison of sedimentary fabrics vs. archaeological fabrics of the mentioned levels and, with this procedure, significant differences arise between both fabric types. These observations, along with other features, reflect to differentiated formation processes that point to the human activity incidence in the taphonomic processes of the archaeological artifacts and the scarce incidence of syn/post-depositional disturbances in this part of Cova Gran archaeological sequence.
\end{abstract}

Keywords: Sedimentary fabrics; archaeological fabrics; trampling, site formation processes; Cova Gran.

Roy Sunyer, Miquel; Roda Gilabert, Xavier; Benito-Calvo, Alfonso; Martínez-Moreno, Jorge; Mora TorCal, Rafael. «Verificando la integridad del registro arqueológico: análisis de fábricas en las unidades arqueológicas del Paleolítico medio/superior de la Cova Gran (Santa Linya, Lleida)». Treballs d’Arqueologia, 2014, núm. 20, p. 55-77. DOI: 10.5565/rev/tda.47 
Miquel Roy Sunyer, Xavier Roda Gilabert, Alfonso Benito-Calvo, Jorge Martínez-Moreno, Rafael Mora Torcal

\section{Introducción}

Determinar la resolución contextual de un yacimiento prehistórico es una cuestión clave para su correcta interpretación. La incidencia de los procesos de formación (site formation processes) y las alteraciones postdeposicionales desempeñan un papel fundamental en la caracterización de un conjunto arqueológico, ya que estos procesos modifican y enmascaran las asociaciones de artefactos y los patrones generados por la actividad antrópica (Butzer, 1982; Schiffer, 1983, 1987). Explicar estas dinámicas es fundamental para evaluar la integridad de las ocupaciones arqueológicas (Dibble et al., 1997; Villa, 2004; Bailey, 2007; Malinsky-Buller et al., 2011), atributo básico - aunque difícil de valorar-a la hora de realizar inferencias de orden espacio/temporal.

En los últimos años se han propuesto varias herramientas con las que detectar la actividad de procesos sin/ postdeposicionales asociados con la formación de los yacimientos (Bertran, 1994; Texier, 2000). Estas se han centrado en el análisis de la dispersión horizontal de los objetos mediante el remontaje (ver diversas aportaciones en Cziesla et al. (ed.), 1990), las técnicas de análisis geoestadístico (ver diversas aportaciones en Kroll and Price (ed.), 1991), o la evaluación de la dispersión vertical de artefactos (Cahen et al., 1979; Villa, 1982, 2004; Bordes, 2002; Mora et al., 2008).

Alternativamente, la aplicación de métodos propios de la geología permite evaluar la incidencia de las transformaciones naturales y culturales (Schiffer, 1983) en la formación de los conjuntos arqueológicos. Uno de estos procedi-
Verificando la integridad del registro arqueológico: análisis de fábricas en las unidades arqueológicas del paleolítico medio/superior de la Cova Gran (Santa Linya, Lleida)

mientos es el análisis de fábricas, técnica originaria de la geología estructural y la sedimentología y que se basa en el análisis estadístico de la orientación y pendiente de los elementos de un depósito tales como los clastos sedimentarios. A partir de estos datos se puede detectar la presencia de patrones de distribución espacial de los componentes sedimentarios y su relación con las geometrías y dinámicas sin/postdeposicionales (Mills, 1983; Benn, 1994; Benn y Ringrose, 2001). Este método se ha aplicado en la interpretación de la formación de los depósitos arqueológicos (Lenoble et al., 2000; Bertran y Lenoble, 2002; Bertran y Texier, 1995; Lenoble y Bertran, 2004; McPherron, 2005; BenitoCalvo et al., 2009; Benito-Calvo y De la Torre, 2011).

«Homogeneidad» y «resolución» son atributos difíciles de definir en un conjunto arqueológico; sin embargo, su caracterización es relevante. Un ejemplo reciente lo proporcionan las implicaciones que derivan de la noción de cambio tecno-cultural detectada durante la «transición» del paleolítico medio/superior. En los últimos años se ha discutido la existencia de posibles interestratificaciones entre Chatelperroniense y Auriñaciense, en sitios como la cueva de Fées o Châtelperron (Gravina et al., 2005; Zilhão et al., 2006; Mellars et al., 2007). En la evaluación del significado de estas "entidades culturales» la discusión de atributos como el grado de homogeneidad de los conjuntos líticos, la escasa resolución de la secuencia estratigráfica o la incidencia de los trabajos de excavación en la recuperación y asignación de estos elementos han sido argumentos centrales. Según Zilhão et al. (2008), esas asociaciones de artefactos que se 
Miquel Roy Sunyer, Xavier Roda Gilabert, Alfonso Benito-Calvo, Jorge Martínez-Moreno, Rafael Mora Torcal

atribuyen a una fase crono-cultural específica son el resultado de la combinación de los procesos de formación del depósito y de las inadecuadas técnicas de recuperación de la muestra. Procesos similares han sido detectados en otras secuencias como Roc de Combe o Le Piage, en las que se habían descrito interestratificaciones similares (Bordes, 2002). Estos factores, que en principio podrían considerarse marginales, cuestionan la validez de estas entidades tecno-tipológicas y, en consecuencia, afectan a la atribución crono-cultural de estos conjuntos. Igualmente, derivan implicaciones sobre la noción de cambio biocultural implícita en el debate sobre la "transición" del paleolítico medio/superior.

Es dentro de esta línea argumental en la que presentamos los resultados del estudio de las fábricas de los niveles arqueológicos de la Cova Gran de Santa Linya (Prepirineo de Lleida), centrándonos en aquellos en los que se identifica este proceso de reemplazo tecno-cultural (Martínez-Moreno et al. 2010, 2012; Mora et al., 2011).

Paralelamente, exponemos los resultados obtenidos en un experimento, al que nos remitimos, dirigido a determinar la incidencia del pisoteo (trampling) como un agente activo en la configuración del registro arqueológico (BenitoCalvo et al., 2011). Este estudio se suma a trabajos anteriores que han descrito los efectos de este proceso a nivel etnoarqueológico o experimental, en los cuales se ha evaluado su incidencia a nivel espacial, o en la identificación de modificaciones sobre los artefactos líticos y restos óseos (entre otros, Stockton 1973; Gifford y Behrensmeyer, 1977; Villa y Courtin, 1983; Gifford-González et al., 1985; Nielsen, 1991; Eren et al., 2010).
Verificando la integridad del registro arqueológico: análisis de fábricas en las unidades arqueológicas del paleolítico medio/superior de la Cova Gran (Santa Linya, Lleida)

Estos trabajos señalan que un pisoteo intenso genera desplazamientos y la reorganización de los artefactos, que permiten identificar posibles zonas de circulación en el interior de una ocupación (Leroi-Gourhan y Brézillon, 1966; Theunissen et al., 1998; Nielsen, 1991). Igualmente, este proceso produce fenómenos de migración vertical que conllevan la mezcla de materiales de diferentes unidades arqueológicas (Villa y Courtin, 1993; Eren et al., 2010).

Pese a la importancia que se confiere a estos procesos tafonómicos, ya que son capaces de promover la reorganización parcial o total del registro arqueológico y de las relaciones espacio-temporales de sus componentes, su incidencia a nivel arqueológico sigue siendo poco conocida. El análisis de fábricas puede convertirse en una técnica relativamente simple con la que evaluar el impacto de los procesos $\sin /$ postdeposicionales en las unidades arqueológicas. Los resultados derivados de este análisis, aplicado en el yacimiento de la Cova Gran, generarán argumentos con los que abordar el significado del cambio tecno-cultural que se relaciona con la aparición del paleolítico superior, fenómeno para el que se han propuesto explicaciones no coincidentes; bien sea el resultado de un proceso de evolución interna anclado en el sustrato musteriense (Bernaldo de Quirós et al., 2008), o en contraposición el resultado de un reemplazo biocultural (Bar-Yosef y Bordes, 2010).

\section{La aplicación del análisis de fábricas en arqueología}

La metodología que estructura el análisis de fábricas en contextos arqueológicos ha 
Miquel Roy Sunyer, Xavier Roda Gilabert, Alfonso Benito-Calvo, Jorge Martínez-Moreno, Rafael Mora Torcal
Verificando la integridad del registro arqueológico: análisis de fábricas en las unidades arqueológicas del paleolítico medio/superior de la Cova Gran (Santa Linya, Lleida) sido formulada por varios autores (Vollmer, 1989; Benn, 1994; Bertran y Teixer, 1995; Bertran et al., 1997; Benn y Ringrose, 2001; Bertran y Lenoble, 2002). Tomando como partida técnicas geológicas basadas en el análisis estadístico de la distribución espacial (Curray, 1956; Woodcock, 1977; Watson, 1965, 1966), estos trabajos han desarrollado varios procedimientos para la visualización e interpretación de los datos.
En el punto de partida de estos análisis, cada elemento (en este caso los artefactos o los clastos de un conjunto arqueológico) es caracterizado en términos de la disposición tridimensional de los ejes morfológicos mayores (cuantificada mediante la medición de orientaciones y pendientes). Diferentes patrones de distribución espacial son revelados a partir del estudio de múltiples índices que se resumen en la tabla 1 .

Tabla 1. Resumen y cálculo de los diferentes índices utilizados por varios autores para el análisis de fábricas

\begin{tabular}{|c|c|c|}
\hline Índice & Características & Explicación \\
\hline \multicolumn{3}{|l|}{ Curray, 1956: } \\
\hline $\mathrm{L}$ & $\begin{array}{l}\text { Rango: } 0 \% \text { : Dispersión máxima } \\
\text { de orientaciones. } \\
\text { 100\%: Todos los ejes apuntan } \\
\text { a la misma dirección. }\end{array}$ & $\begin{array}{l}\text { Adición de las direcciones de los ejes mayores } \\
\text { que varían de } 0^{\circ}-180^{\circ} \text {. }\end{array}$ \\
\hline$p(\mathrm{~L})$ & $\begin{array}{l}\text { Rango: } 0-100 \% \text { (probabilidad) } \\
\text { Condición: Únicamente para } \\
\text { poblaciones no plurimodales. }\end{array}$ & $\begin{array}{l}\text { Probabilidad de obtener } p \text { mayores que } \mathrm{L} \\
\text { por combinación aleatoria de fases. }\end{array}$ \\
\hline Vector medio & $\begin{array}{l}\text { Rango: Vector (orientación, ángulo } \\
\text { de buzamiento y módulo). } \\
\text { Obtención: Suma de vectores / } N\end{array}$ & $\begin{array}{l}\text { El módulo tiene valores máximos cuando la } \\
\text { dispersión es mínima y valores mínimos } \\
\text { cuando la dispersión es máxima. }\end{array}$ \\
\hline $\mathrm{R} \%$ & $\begin{array}{l}\text { Rango: } 0-100 \% \\
\text { Obtención: Módulo vector medio } \\
\text { / } N\end{array}$ & $\begin{array}{l}\text { Vector medio normalizado por } n \text {. Constituye } \\
\text { el grado de orientación preferencial. }\end{array}$ \\
\hline
\end{tabular}

Vectores propios (Vollmer 1989, Benn 1994, Benn y Rongrose 2001, Lenoble y Bertran 2004, McPherron 2005, Lenoble et al. 2008):

Vectores Rango: Vectores ortogonales Vectores ortogonales entre sí. El plano propios. (orientación, ángulo de buzamiento V1-V2 corresponde al plano preferencial de V1, V2 y V3: y módulo)

Valores propios Rango: $0-1$

S1, S2 y S3: Obtención: Vectores propios normalizados por sus módulos. la fábrica.

Tensor de orientaciones que define la forma de la fábrica.

Los módulos reflejan el grado de agrupamiento del conjunto.

Fábricas isotrópicas: $\mathrm{S} 1=\mathrm{S} 2=\mathrm{S} 3$

Fábricas planares: $\mathrm{S} 1=\mathrm{S} 2>>\mathrm{S} 3$

Fábricas lineales: $\mathrm{S} 1>>\mathrm{S} 2=\mathrm{S} 3$ 
Miquel Roy Sunyer, Xavier Roda Gilabert, Alfonso Benito-Calvo, Jorge Martínez-Moreno, Rafael Mora Torcal
Verificando la integridad del registro arqueológico: análisis de fábricas en las unidades arqueológicas del paleolítico medio/superior de la Cova Gran (Santa Linya, Lleida)

\begin{tabular}{|c|c|c|c|}
\hline \multicolumn{4}{|c|}{ Woodcock 1977: } \\
\hline $\begin{array}{l}\text { Ratios de } \\
\text { valores propios } \\
\text { r1 y r2: }\end{array}$ & $\begin{array}{l}\text { Obtención: } \\
\mathrm{r} 1=\ln (\mathrm{S} 1 / \mathrm{S} 2) \\
\mathrm{r} 2=\ln (\mathrm{S} 2 / \mathrm{S} 3)\end{array}$ & $\begin{array}{l}\text { Resumen la fábrica } \\
\text { de un conjunto }\end{array}$ & \multirow{2}{*}{$\begin{array}{l}\text { Diagramas biaxiales } \\
\text { ortogonales donde K es } \\
\text { la bisectriz que permite } \\
\text { diferenciar las fábricas } \\
\text { planares de las lineares }\end{array}$} \\
\hline K: & $\mathrm{K}=\mathrm{r} 1 / \mathrm{r} 2$ & $\begin{array}{l}0<\mathrm{K}<1 \text { : F. planares. } \\
1<\mathrm{K}<\infty \text { : F. lineares. }\end{array}$ & \\
\hline \multicolumn{4}{|c|}{ Woodcock y Naylor, 1983: } \\
\hline $\begin{array}{l}\text { Intiensidad de } \\
\text { fábrica, C: }\end{array}$ & Obtención: $\mathrm{C}=\ln (\mathrm{S} 1 / \mathrm{S} 3)$ & $\begin{array}{l}\text { Cuanto mayor es C, } \\
\text { más lejos quedan de } \\
\text { las fábricas } \\
\text { isotrópicas. }\end{array}$ & $\begin{array}{l}\text { Diagramas biaxiales } \\
\text { ortogonales }\end{array}$ \\
\hline \multicolumn{4}{|l|}{ Benn, 1994: } \\
\hline $\begin{array}{l}\text { Índices de } \\
\text { isotropía y } \\
\text { elongación: }\end{array}$ & $\begin{array}{l}\text { Obtención: } \\
\mathrm{l}=\mathrm{S} 3 / \mathrm{S} 1 \\
\mathrm{E}=1-(\mathrm{S} 2 / \mathrm{S} 1)\end{array}$ & $\begin{array}{l}\text { Refleja la variación } \\
\text { continua de la forma } \\
\text { de la fábrica, } \\
\text { delimitada por } \\
\text { los vértices } \\
\text { correspondientes } \\
\text { a las fábricas } \\
\text { isotrópicas, planares } \\
\text { y lineares. }\end{array}$ & $\begin{array}{l}\text { Diagrama triangular } \\
\text { de Sneed \& Folk. Los } \\
\text { vértices corresponden } \\
\text { a los términos máximos } \\
\text { de isotropía, planaridad } \\
\text { y linealidad. }\end{array}$ \\
\hline $\begin{array}{l}\text { Índices planar } \\
\text { y Cluster- } \\
\text { Girdle: }\end{array}$ & $\begin{array}{l}\text { Rango: } 0 \text { (fábricas planares) }-1 \\
\text { (fábricas lineares). } \\
\text { Obtención: } \\
\mathrm{F}=\mathrm{S} 3 / \mathrm{S} 2 \\
\mathrm{CGI}=\mathrm{S} 1-\mathrm{S} 2 / \mathrm{S} 1-\mathrm{S} 3\end{array}$ & & \\
\hline \multicolumn{4}{|c|}{ Vollmer, 1989: } \\
\hline $\begin{array}{l}\text { Índices planar } \\
\text { o gridle }(\mathrm{G}) \text {, } \\
\text { linear o } \\
\text { cluster }(\mathrm{C}), \mathrm{e} \\
\text { isotrópico }(\mathrm{U}) \\
\end{array}$ & $\begin{array}{l}\text { Obtención: } \\
\mathrm{G}=2(\mathrm{~S} 2-\mathrm{S} 3) / \mathrm{n} \\
\mathrm{C}=(\mathrm{S} 1-\mathrm{S} 2) / \mathrm{n} \\
\mathrm{U}=3(\mathrm{~S} 3 / \mathrm{n})\end{array}$ & $\begin{array}{l}\text { Condición: } \\
\mathrm{G}+\mathrm{C}+\mathrm{U}=1\end{array}$ & Diagrama ternario \\
\hline
\end{tabular}

Las primeras aproximaciones al estudio de fábricas fueron llevadas a cabo por Curray (1956). Los índices L, p(de L) y $\mathrm{R} \%$ permiten caracterizar la distribución de un conjunto en términos del grado de dispersión de las direcciones de los ejes principales. El vector magnitud «L», es un índice bidimensional que indica la extensión del vector resultante de la adición de las direcciones de los ejes mayores que varían entre $0^{\circ}-180^{\circ}$. L se expresa en tanto por ciento y sus valores van desde 0\% (la dirección de los ejes presenta una dispersión máxima) hasta 100\% (todos los ejes apuntan en la misma dirección). Este índice puede ser usado, siempre y cuando la distribución no sea plurimodal, para estimar la probabilidad $(p)$ 
Miquel Roy Sunyer, Xavier Roda Gilabert, Alfonso Benito-Calvo, Jorge Martínez-Moreno, Rafael Mora Torcal

de obtener un valor mayor que L por pura combinación aleatoria de fases (Curray, 1956).

Si consideramos que la disposición de los objetos indica el sentido de $n$ vectores, las muestras también pueden ser caracterizadas calculando el vector medio (orientación, ángulo de buzamiento y módulo). A su vez, el módulo de dicho vector constituye un índice de dispersión, con valores máximos cuando la dispersión es mínima y valores mínimos cuando la dispersión es máxima, ya que estos vectores se suman o se anulan. Este módulo, normalizado por el tamańo de muestra, se representa en tanto por ciento, y constituye el grado de orientación preferencial $(R \%)$.

Sin embargo, el procedimiento más utilizado es el método de los vectores propios (Vollmer, 1989; Benn, 1994; Benn y Rongrose, 2001; Lenoble y Bertran, 2004; McPherron, 2005; Lenoble et al., 2008). Esta técnica simplifica el conjunto de medidas en un tensor de orientaciones que define la forma de la fábrica, y que se compone a partir de tres vectores propios (V1, V2 y V3) ortogonales entre sí. La orientación dominante es representada por V1 que junto a V2 forma el plano preferencial de la fábrica, mientras que $\mathrm{V} 3$ es ortogonal a dicho plano. El grado de agrupamiento de la población en relación con los vectores propios es reflejado por sus módulos. Los vectores propios, normalizados por sus módulos, dan lugar a los llamados valores propios S1, S2 y S3. Las relaciones entre valores propios hacen posible diferenciar entre fábricas isotrópicas $(\mathrm{S} 1=\mathrm{S} 2=\mathrm{S} 3)$, planares $(\mathrm{S} 1=\mathrm{S} 2>>\mathrm{S} 3)$ y lineares $(\mathrm{S} 1>\mathrm{S} 2=\mathrm{S} 3)$.

A partir de los vectores propios se pueden calcular múltiples índices que
Verificando la integridad del registro arqueológico: análisis de fábricas en las unidades arqueológicas del paleolítico medio/superior de la Cova Gran (Santa Linya, Lleida) resumen numéricamente la fábrica de un conjunto. Woodcock (1977) define las ratios de valores propios $\mathrm{r} 1=\ln (\mathrm{S} 1 / \mathrm{S} 2)$ y $\mathrm{r} 2=\ln (\mathrm{S} 2 / \mathrm{S} 3)$, que son proyectados en diagramas biaxiales y ortogonales, donde el índice $\mathrm{K}=\mathrm{r} 1 / \mathrm{r} 2$ representa la bisectriz que permite diferenciar las fábricas planares $(0<\mathrm{K}<1)$ de las lineares $(1<\mathrm{K}<\infty)$. Utilizando este método, Woodcock y Naylor (1983) definen el parámetro «intensidad de fábrica» o $C(\mathrm{C}=\ln (\mathrm{S} 1)$ S3). Cuanto mayor es el parámetro C, más lejos quedan los valores del centro del diagrama donde se encuentran las fábricas isotrópicas.

Otra representación utilizada para la proyección de fábricas sedimentarias es la propuesta por Benn (1994) al definir los índices de isotropía $(1=S 3 / S 1)$ y de elongación $(E=1-(\mathrm{S} 2 / \mathrm{S} 1)$. Estos son proyectados en un diagrama triangular de Sneed y Folk, donde se refleja la variación continua de la forma de la fábrica, delimitada por los vértices correspondientes a las fábricas isotrópicas, planares y lineares. Otros índices propuestos por Benn (1994) relacionan el índice planar $(\mathrm{F}=\mathrm{S} 3 / \mathrm{S} 2)$ o el índice cluster-girdle $(\mathrm{CGI}=\mathrm{S} 1-\mathrm{S} 2 / \mathrm{S} 1-\mathrm{S} 3)$, que varían entre 0 (fábricas planares) y 1 (fábricas lineares). Por su parte, Vollmer (1989) propone la proyección triangular de las fábricas a partir de los índices planar o girdle $(\mathrm{G}=2(\mathrm{~S} 2-\mathrm{S} 3) / \mathrm{n})$, linear o cluster $(\mathrm{C}=(\mathrm{S} 1-\mathrm{S} 2) / \mathrm{n})$ e isotrópico o uniforme $(U=3(S 3 / n))$, que entre ellos suman 1 y se representan sobre un diagrama ternario.

En este artículo aplicaremos estos índices a fin de evaluar dos aspectos. Por un lado, se analizan separadamente las fábricas sedimentarias y arqueológicas en los niveles relacionados con la transición Paleolítico medio/superior en la Cova 
Miquel Roy Sunyer, Xavier Roda Gilabert, Alfonso Benito-Calvo, Jorge Martínez-Moreno, Rafael Mora Torcal
Verificando la integridad del registro arqueológico: análisis de fábricas en las unidades arqueológicas del paleolítico medio/superior de la Cova Gran (Santa Linya, Lleida)
Gran, aportando nuevos datos a los previamente discutidos en Benito-Calvo et al. (2009). Por el otro, los resultados son comparados con los obtenidos en un experimento que desarrollamos con la intención de valorar la incidencia del pisoteo en las fábricas de los conjuntos arqueológicos del yacimiento (BenitoCalvo et al., 2011).

\section{Contexto geográfico y geológico}

La Cova Gran de Santa Linya $(X=318541$, $\mathrm{Y}=4643877, \mathrm{H} 31, \mathrm{ETRS} 89,385 \mathrm{~m}$ snm) es un abrigo de $80 \times 40$ m de planta protegido por una visera caliza de 30 $\mathrm{m}$ de altura (figura 1). El yacimiento se ubica en el fondo de un estrecho valle en el que se encaja el torrente de Sant
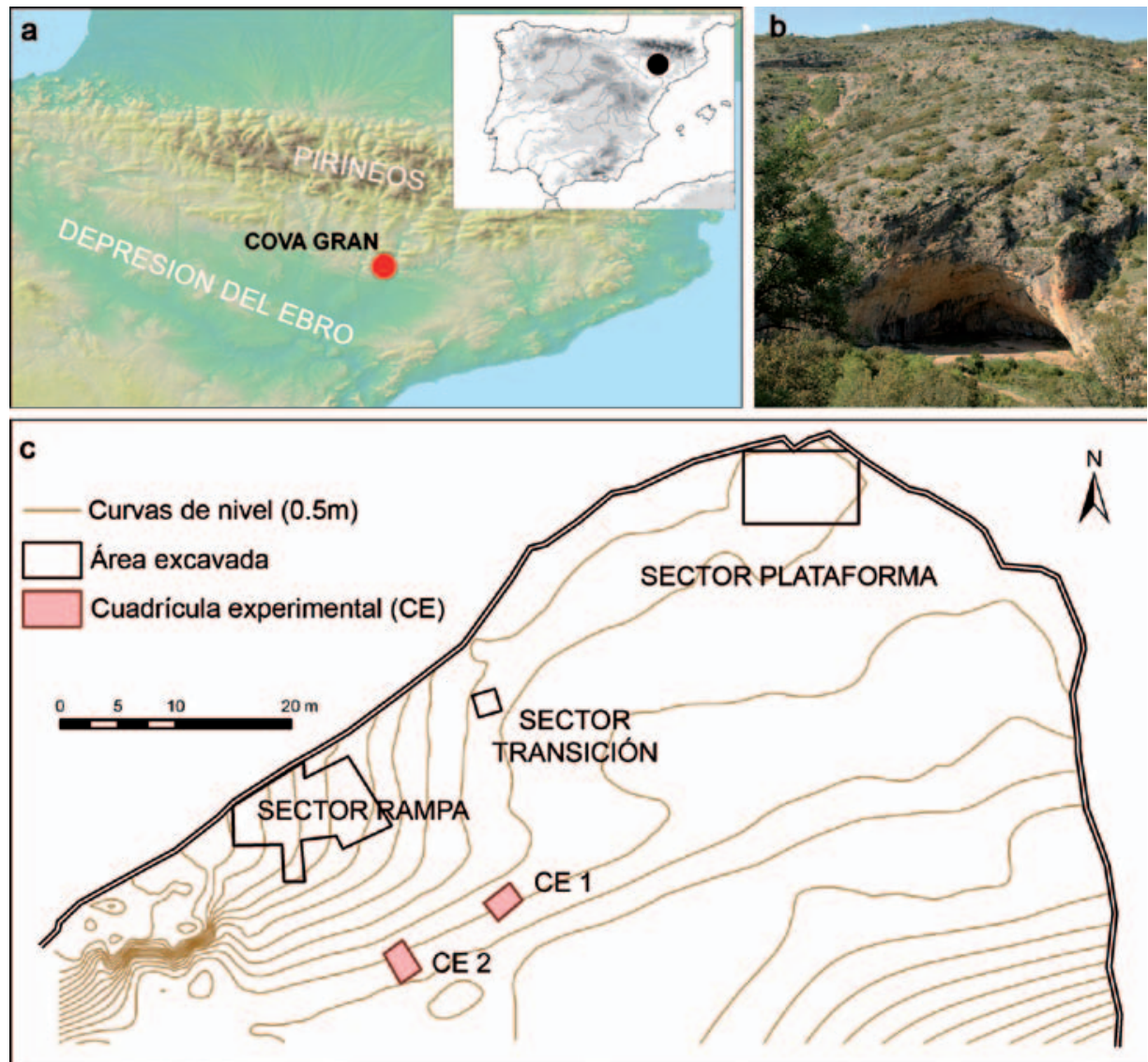

Figura 1. Área de estudio. a) Localización geográfica dentro de la Península Ibérica; b) Vista general del yacimiento; c) Planta del yacimiento con la ubicación de las zonas de excavación y las cuadrículas experimentales. 
Miquel Roy Sunyer, Xavier Roda Gilabert, Alfonso Benito-Calvo, Jorge Martínez-Moreno, Rafael Mora Torcal

Miquel, curso que desagua en el Noguera Pallaresa. Este torrente discurre a lo largo del contacto entre las calizas campanienses (cretácico superior) y las arcillas, yesos y doleritas del Keuper (triásico superior). Aunque actualmente no transporta agua con regularidad, es posible que la cavidad fuera afectada por crecidas de su cauce, tal y como se ha señalado en otros cursos de la cuenca hidrográfica en la que se encuadra el torrente (Rico Herrero, 2004).

Geográficamente se integra en el segundo arco de las Sierras Marginales Catalanas (SMC). Las SMC conforman el contacto entre las dos unidades morfoestructurales y biogeográficas que caracterizan el tercio septentrional de la Península Ibérica: la Depresión del Ebro y el arco Pirenaico (figura 1). Esta unidad geológica se configura por un conjunto de mantos de cobertera mesozoica y cenozoica que se suceden de norte a sur, dando lugar a un conjunto de estructuras tectónicas con rellenos cuaternarios (Pocoví, 1978; Peña, 1983).

A nivel regional, destaca el papel de los ríos Noguera Pallaresa y Noguera Ribagorzana que han modelado un paisaje abrupto con fondos de valle encajados pero de fácil acceso y de alta conectividad entre ellos a partir de la red fluvial. El sustrato calizo de estas sierras configura sistemas kársticos poco desarrollados que propician la formación de cuevas y abrigos utilizados regularmente hasta prácticamente hoy en día. Algunos de ellos conservan indicadores de su ocupación durante el pleistoceno superior y el holoceno (Pizarro et al., 2013). Aunque la Cova Gran se localiza en un pequeño valle transversal, su posición permite un fácil acceso a los valles interiores del Pirineo a partir de los escarpes
Verificando la integridad del registro arqueológico: análisis de fábricas en las unidades arqueológicas del paleolítico medio/superior de la Cova Gran (Santa Linya, Lleida) excavados por el Noguera Ribagorçana y el Noguera Pallaresa en la sierra del Montsec. Igualmente, su cercanía al curso medio del Segre permite una rápida conexión con la Depresión del Ebro.

\section{Secuencia estratigráfica y registro material}

Debido a la gran extensión del yacimiento, no se dispone de una secuencia estratigráfica completa. Hasta el momento se han diferenciado dos secuencias estratigráficas parciales que no se han podido conectar físicamente. Al oeste del abrigo se preserva un depósito gravitacional que se apoya sobre grandes bloques y que denominamos sector Rampa (R), compuesto por clastos calizos y matriz areno-arcillosa. Este cuerpo sedimentario, que alcanza una altura de $+9 \mathrm{~m}$ de altura relativa respecto al lecho del arroyo, desciende hacia el E — sector transición (T) - conectando con una plataforma $(\mathrm{P})$ horizontal localizada a $+3 \mathrm{~m}$ del torrente de Sant Miquel. En el sector P se identifican sedimentos arenosos recientes que se extienden hacia un plano inferior (LP) sobre el que incide actualmente el arroyo (figura 1) (Mora et al., 2011).

La secuencia más antigua identificada en el depósito se sitúa en el sector Rampa, en la que se ha excavado una secuencia de $3 \mathrm{~m}$ de espesor sin alcanzarse el sustrato, y en la que se diferencian dos unidades litoestratigráficas: unidad inferior -S1-, y unidad superior -497(figura 2).

La unidad S1 está formada por derrubios autóctonos que se articulan en varios tramos de contacto gradual separado por distintas caídas de bloques de tamaño métrico. En conjunto son sedi- 


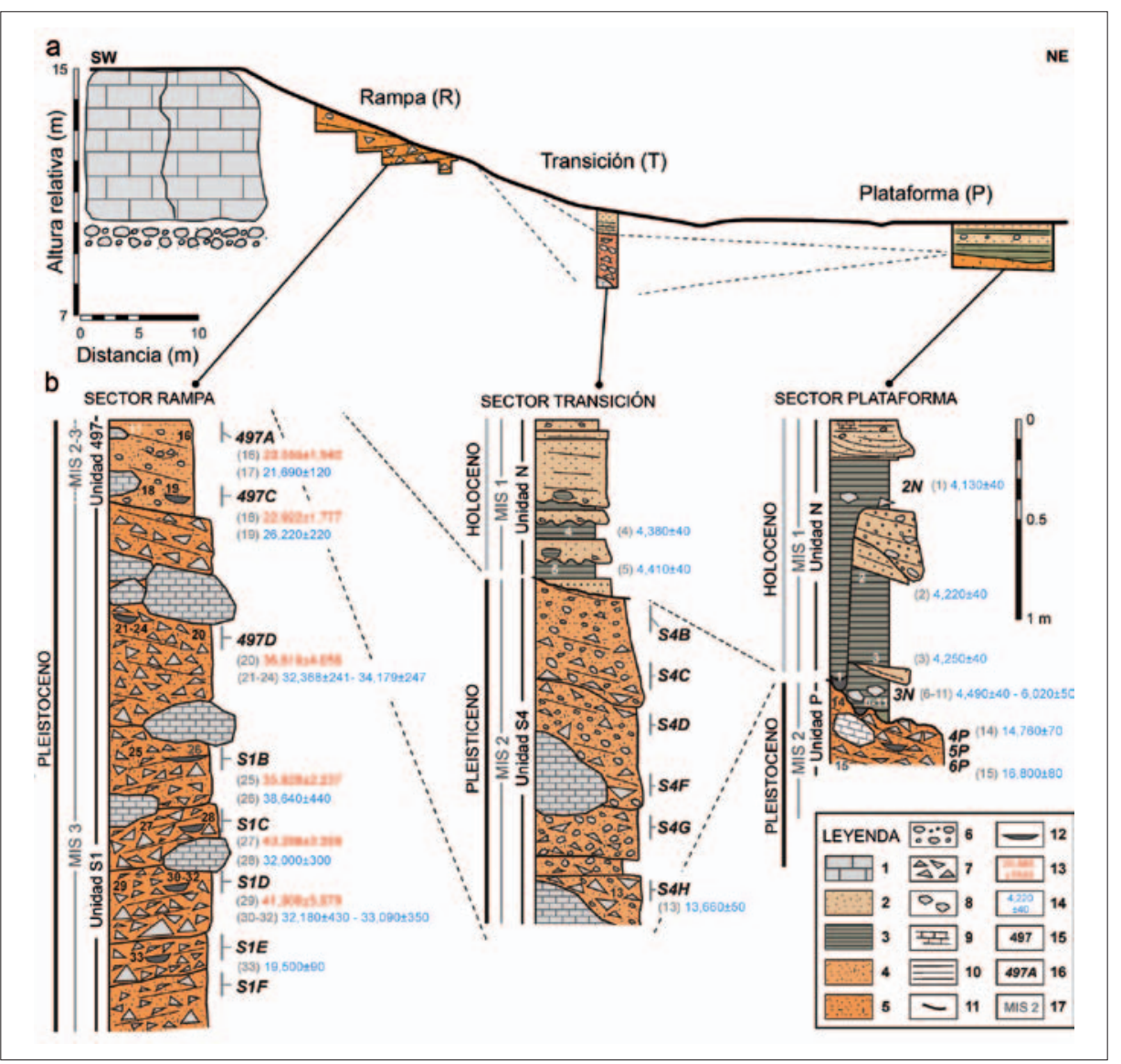

Figura 2. Secuencia estratigráfica de la Cova Gran; a) Transecto estratigráfico del yacimiento; b) Secuencia estratigráfica, arqueológica y cronométrica de la Cova Gran por sectores. Leyenda: 1: Bloques de caliza; 2: Arenas; 3: Fumiers; 4: Arcillas y limos; 5: Arenas, limos y sales; 6: Clastos redondeados y subredondeados; 7: Clastos angulares y subangulares; 8: Bloques arqueológicos; 9: Carbonatación; 10: Líneas de estratificación; 11: Discontinuidad; 12: Hogar; 13: Datación TL; 14: Datación 14C AMS; 15: Unidad estratigráfica; 16: Nivel arqueológico; 17: estadio isotópico marino (MIS) (ver Mora et al., 2011).

mentos angulares o muy angulares de tamaños medios y mal clasificados. La matriz es escasa $(<20 \%)$ y está integrada por arenas limo-arcillosas que aumentan de techo a muro. La fracción fina está compuesta principalmente por calcita y dolomita, que decrecen hacia techo coincidiendo con el aumento de minerales silíceos (cuarzo, albita, ilita y clinocloro) y la aparición de yeso (Benito-Calvo et al., 2009). En la configuración de esta unidad se definen dos áreas sedimentoló- 
Miquel Roy Sunyer, Xavier Roda Gilabert, Alfonso Benito-Calvo, Jorge Martínez-Moreno, Rafael Mora Torcal

gicamente diferenciadas. Los tramos basales excavados en la zona $\mathrm{E}$ de la rampa presentan una pendiente W-SW que progresivamente se vuelve horizontal. Los tramos superiores, situados al W adquieren una pendiente en sentido E-NE. Se han reconocido cinco ocupaciones correspondientes al paleolítico medio (S1F, S1E, S1D, S1C y S1B), cada una de ellas con un espesor medio de entre 10 y $15 \mathrm{~cm}$. Estos niveles contienen abundante industria lítica elaborada en sílex y rocas metamórficas, y restos de fauna, entre los que se identifica Stephanorbinus sp., Equus caballus, Equs cfr. hydruntinus, Cervus elaphus, Bos sp., Capra pirenaica y Oryctolagus cuniculus. A techo de esta unidad se ha excavado el nivel arqueológico 497D que tiene un espesor medio de 5-10 cm. Los atributos tecno-tipológicos de la industria lítica recuperada (làminas, laminitas rectilíneas y lascas) permiten su adscripción al paleolítico superior inicial (MartínezMoreno et al., 2010, 2012).

Sobre la unidad S1 se apoya el cuerpo sedimentario 497. Este se dispone con una inclinación de $12^{\circ}$ en dirección $\mathrm{N} 70^{\circ}$ $\mathrm{E}$, compuesto por materiales autóctonos asociados a procesos de arrollada difusa, tal y como señala la presencia de gravas finas y muy finas redondeadas y subredondeadas englobadas en una abundante matriz de arenas lutíticas (40\%). Entre los minerales de la matriz domina la calcita y el cuarzo a partes iguales, con presencia minoritaria de albita, ilita y clinocloro (Benito-Calvo et al., 2009). Este lecho sedimentario contiene las unidades arqueológicas 497C y 497A, que presentan unas características tecno-tipológicas similares a las de la unidad 497D. La fauna recuperada en estos niveles incluye Equus caballus, Cervus elaphus y Capra
Verificando la integridad del registro arqueológico: análisis de fábricas en las unidades arqueológicas del paleolítico medio/superior de la Cova Gran (Santa Linya, Lleida)

pirenaica. En todos los niveles de paleolítico superior se han recuperado ornamentos marinos, siendo la especie más abundante Nassarius pygmaed.

En los sectores $\mathrm{T}$ y $\mathrm{P}$ aparecen niveles sedimentarios que se adscriben al final del pleistoceno superior y holoceno. Estas secuencias se apoyan directamente a partir de un contacto discordante sobre los depósitos pleistocenos y señalan un paleorrelieve que erosiona parcialmente los sedimentos infrayacentes. Estos depósitos contienen unidades arqueológicas atribuidas al magdaleniense antiguo, magdaleniense medio-superior y mesolítico junto a varias ocupaciones neolíticas. En estas últimas se han identificado estructuras como hogares, fosas de almacenaje y agujeros de poste que se combinan con facies de fumier, entre los que se intercalan eventos tractivos de arenas y gravas (Mora et al., 2011).

\section{Metodología}

Proponemos analizar de forma independiente las orientaciones preferenciales y pendientes de clastos y artefactos con el fin de evaluar si existen diferencias entre las fábricas sedimentarias y arqueológicas (figuras 3a, $3 \mathrm{~b}$ y 4 ). Para este análisis se han calculado los índices $\mathrm{K}$ y $\mathrm{C}$ (Woodcock, 1977; Woodcock y Naylor, 1983), los vectores propios (V1, V2 y V3) y el índice de orientación preferencial (R\%). Debido a sus características ternarias, se ha aplicado el triángulo de Vollmer (SpheriStat 3). Para el cálculo se han incluido los datos recuperados hasta 2011 ( $n=1097)$, lo que permite su comparación con los resultados ya publicados para estos niveles (tablas 1 y 2) (Benito-Calvo et al., 2009, 2011). 
Miquel Roy Sunyer, Xavier Roda Gilabert, Alfonso Benito-Calvo, Jorge Martínez-Moreno, Rafael Mora Torcal
Verificando la integridad del registro arqueológico: análisis de fábricas en las unidades arqueológicas del paleolítico medio/superior de la Cova Gran (Santa Linya, Lleida)
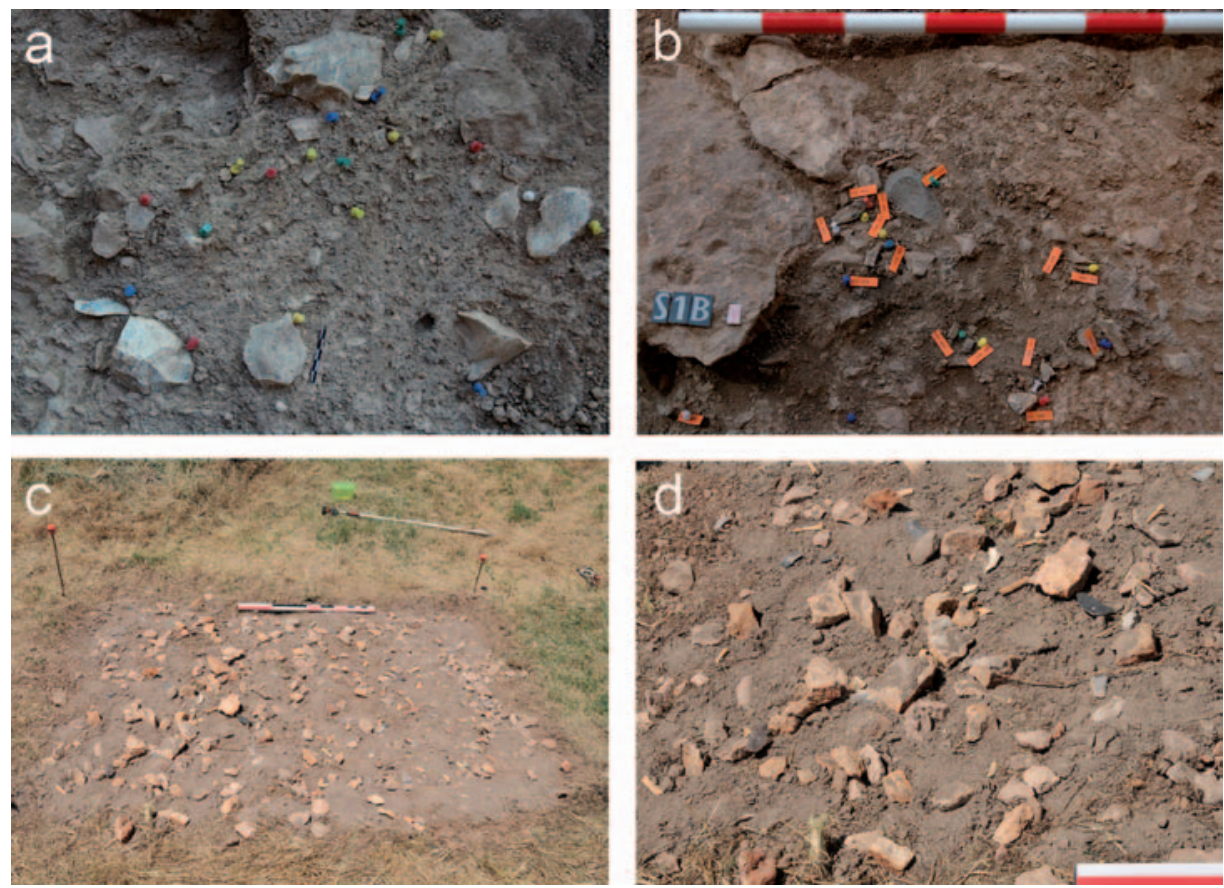

Figura 3. Materiales arqueológicos y clastos procedentes de la excavación y las cuadrículas experimentales. a) Acumulación procedente del nivel 497C; b) acumulación del nivel S1B; c) Vista general de la cuadrícula experimental 1 (CE 1); d) Detalle de los materiales de la CE 1.

La posición tridimensional de los artefactos arqueológicos fue registrada con la ayuda de una estación total, mientras que los datos de las fábricas (orientación y ángulo de buzamiento) se tomaron con brújula y clinómetro (figura 4). Estos datos se refieren a los ejes mayores de objetos superiores a $2 \mathrm{~cm}$ (Kjaer and Krüger, 1998) y con índices de elongación superiores a 1,6. Las medidas han sido directamente representadas en histogramas angulares (orientación) o proyecciones estereográficas (orientación y ángulo de buzamiento). Siguiendo la indicación de Lenoble y Bertran (2004), únicamente se analizan unidades arqueológicas que disponen más de 50 efectivos.

En un trabajo anterior se llevó a cabo un programa experimental con el fin de valorar la incidencia del pisoteo sobre las fábricas arqueológica (BenitoCalvo et al., 2011). Se crearon dos cuadrículas experimentales $(3 \times 2 \mathrm{~m})$ en las que se depositaron sedimentos autóctonos del yacimiento, para simular las características sedimentarias del sector Rampa, junto a reproducciones de materiales arqueológicos (restos líticos y óseos) de los cuales se tomó la posición, orientación y pendiente (figuras $3 \mathrm{c} y$ 3d). Tras someter ambas celdas a pisoteo 


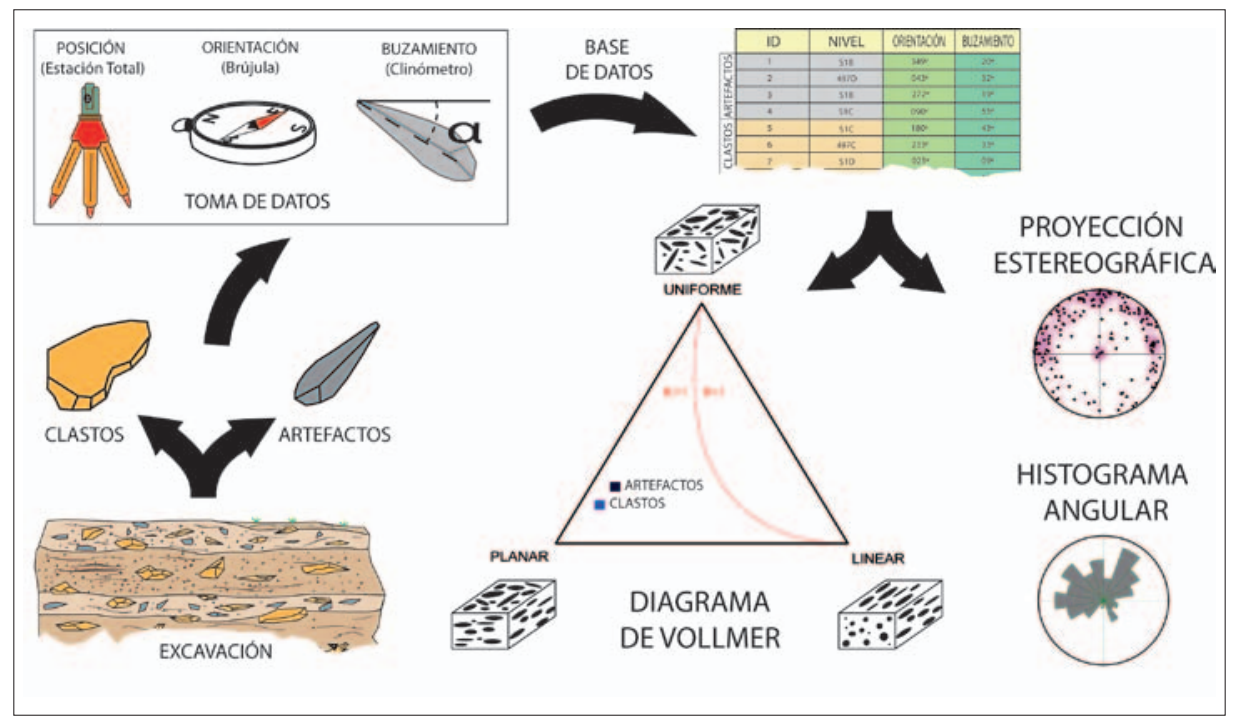

Figura 4. Resumen de la metodología empleada para la obtención y tratamiento de los datos de las fábricas sedimentarias y arqueológicas.

continuado durante seis semanas, fueron excavadas con la misma metodología aplicada en el resto de la excavación. La posición, orientación y pendiente de cada pieza fueron tomadas con estación total, brújula y clinómetro. Por medio de ArcGIS 10 y SpheriStat 3, se calcularon el desplazamiento horizontal y vertical de los objetos y la distribución direccional de estos conjuntos con el fin de conocer los cambios sufridos en la distribución de los artefactos. Las características del experimento han sido difundidas en Benito-Calvo et al. (2011).

\section{Resultados}

El estudio de las fábricas sedimentarias y arqueológicas de las unidades arqueológicas de la Cova Gran permite cuantificar los parámetros referidos anteriormente en el apartado de metodología (tabla 2). Se dispone de datos sobre la orientación y la pendiente de los clastos de las unidades 497A, 497C, 497D, S1B y S1C, y de los artefactos de los niveles 497C, 497D, S1B y S1C que globalmente constituyen un conjunto de $n=1097$ que incrementa sustancialmente las poblaciones analizadas en contribuciones anteriores (Benito-Calvo et al., 2009, 2011).

\subsection{Fábricas sedimentarias}

Los resultados obtenidos a partir del cálculo de índices de las fábricas sedimentarias indican el predominio de las fábricas planares (índice planar o girdle $0,59<\mathrm{G}$ $<0,79$ ). El grado de orientación preferencial descarta las fábricas lineares $(25,6$ $<\mathrm{R} \%<42,1)$. Del mismo modo, las 
Miquel Roy Sunyer, Xavier Roda Gilabert, Alfonso Benito-Calvo, Jorge Martínez-Moreno, Rafael Mora Torcal
Verificando la integridad del registro arqueológico: análisis de fábricas en las unidades arqueológicas del paleolítico medio/superior de la Cova Gran (Santa Linya, Lleida)

Tabla 2. Índices calculados para el análisis de las fábricas de los artefactos y los clastos, n) Tamaño de la muestra; V1, V2, V3) Vectores propios calculados para la muestra, O) Orientación, B) Buzamiento; V. Pr) Valores propios; Índice de las fabricas Vollmer, C) Cluster, G) Gridle, U) Uniforme; Índice de Woodcock, K) Índice $K$ de Woodcock, C) Intensidad de la fábrica, R\%) módulo del vector resultante expresado en \%. Desviación estándar $(\sigma)$

\begin{tabular}{|c|c|c|c|c|c|c|c|c|c|c|c|c|c|c|c|c|c|}
\hline & & V1 & & V2 & & V3 & & & Voll & mer & & & & Wood & dcock & & \\
\hline Nivel $n$ & $\mathrm{O}$ & B V.Pr & $\mathrm{O}$ & B V.Pr & $\mathrm{O}$ & B V.Pr & $\mathrm{C}$ & $\sigma$ & $\mathrm{G}$ & $\sigma$ & $\mathrm{U}$ & $\sigma$ & K & $\sigma$ & $\mathrm{C}$ & $\sigma$ & $\mathrm{R} \%$ \\
\hline \multicolumn{18}{|l|}{ Artefactos } \\
\hline $497 \mathrm{C} 109$ & 57 & 555,05 & 3261 & 1242,67 & 168 & $\begin{array}{llll}71,28 & 11\end{array}$ & 0,114 & 0,007 & 0,576 & 0,576 & 0,310 & 0,018 & 0,192 & 0,009 & 1,585 & 0,029 & 26,4 \\
\hline 497D 178 & 298 & 977,11 & 301 & 1273,89 & 1727 & 7527,00 & 0,018 & 0,005 & 0,527 & 0,011 & 0,455 & 0,011 & 0,042 & 0,006 & 1,049 & 0,012 & 35,2 \\
\hline S1B & 54 & 356,14 & 3231 & 1451,75 & 157 & 7520,11 & 0,034 & 0,006 & 0,494 & 0,014 & 0,471 & 0,015 & 0,086 & 0,009 & 1,027 & 0,016 & 27,5 \\
\hline S1C & 44 & 365,35 & 314 & 258,72 & 194 & $87 \quad 14,93$ & 0,048 & 0,006 & 0,630 & 0,014 & 0,322 & 0,015 & 0,078 & 0,005 & 1,476 & 0,023 & 11,2 \\
\hline \multicolumn{18}{|l|}{ Clastos } \\
\hline 497A 81 & 321 & 1139,51 & 2991 & 1232,73 & 162 & $\begin{array}{ll}73 & 8,77\end{array}$ & 0,084 & 0,01 & 0,592 & 0,01 & 0,325 & 0,025 & 0,143 & 0,011 & 1,506 & 0,039 & 37,1 \\
\hline 497C 66 & 286 & 134,12 & 161 & 1128,77 & 192 & $79 \quad 3,11$ & 0,081 & 0,011 & 0,778 & 0,029 & 0,141 & 0,032 & 0,077 & 0,007 & 2,395 & 0,112 & 41,5 \\
\hline 497D 126 & 3351 & 1064,00 & 66 & 455,00 & 176 & $79 \quad 6,60$ & 0,071 & 0,006 & 0,771 & 0,016 & 0,158 & 0,018 & 0,071 & 0,004 & 2,267 & 0,056 & 42,1 \\
\hline S1B & 260 & 565,00 & 3511 & 1056,00 & 144 & 7910,00 & 0,069 & 0,005 & 0,699 & 0,014 & 0,232 & 0,015 & 0,087 & 0,004 & 1,856 & 0,032 & 31,8 \\
\hline S1C & 104 & 636,00 & 13 & 728,00 & 233 & $81 \quad 2,70$ & 0,120 & 0,011 & 0,760 & 0,03 & 0,120 & 0,032 & 0,107 & 0,009 & 2,606 & 0,133 & 25,6 \\
\hline
\end{tabular}

proyecciones estereográficas muestran una distribución planar de los clastos (tabla 2 y figura 5). La unidad superior, que contiene los niveles 497A y 497C, presenta una orientación del vector propio principal V1 hacia el NE, siguiendo la inclinación del cuerpo sedimentario, con un bajo ángulo de buzamiento, mientras que los clastos de las unidades 497D, S1B y SIC, que se encuentran en la unidad inferior, presentan bajos ángulos de buzamiento y sin orientación preferencial concreta de V1 (tabla 2).

En todas las unidades se documentan índices $\mathrm{K} \approx 0$ que sitúan el complejo en el campo de las fábricas planares $(0<$ $\mathrm{K}<1$ ). Los valores de intensidad de fábrica (C) varían de moderados a altos $(1,506<\mathrm{C}<2,606)$ (tabla 2$)$. Todos los conjuntos sedimentarios estudiados presentan una distribución planar, situándose próximos al vértice de las fábricas planares, siguiendo el eje planar-unifor- me del diagrama de Vollmer (1989) (figura 6). Estos datos indican la ausencia de movimientos postdeposicionales que causen la alineación de clastos. Esta forma planar de las fábricas se ajusta a los patrones establecidos para depósitos gravitacionales (Lenoble et al., 2008), lo que descarta que el torrente de Sant Miquel haya influido sobre los cuerpos sedimentarios del sector Rampa.

En los depósitos gravitacionales es frecuente una cierta componente isotrópica que viene magnificada por la pendiente y la rugosidad del terreno (Benn, 1994; Mills, 1983; Bertran et al., 1997). En la Cova Gran, el bajo grado de isotropía puede ser debido a la leve pendiente del depósito. Aunque la orientación de los vectores propios principales (V1) debería reflejar la orientación e inclinación de los diferentes cuerpos sedimentarios, al tratarse de un depósito con morfología irregular es de esperar 
Miquel Roy Sunyer, Xavier Roda Gilabert, Alfonso Benito-Calvo, Jorge Martínez-Moreno, Rafael Mora Torcal

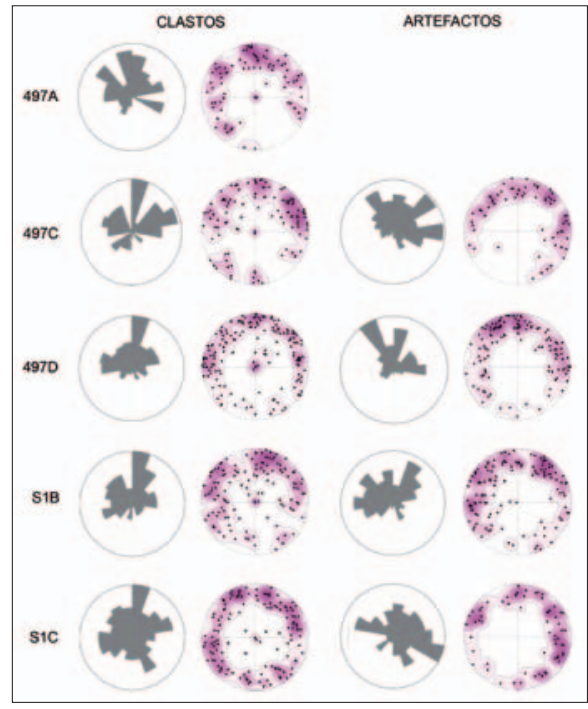

Figura 5. Proyecciones estereográficas y densidad de distribución de los datos del azimut y buzamiento para los clastos y los artefactos de los niveles 497A, 497C, 497D, S1B y S1C.

que la orientación de los estratos varíe en cada punto. Este fenómeno se ve reflejado en la orientación de V1 de las distintas unidades del cuerpo S1 (497D, S1B y S1C), excavadas en una mayor extensión. En cambio, los valores de V1 en la unidad 497 (497A y 497C) reflejan la orientación preferencial de los clastos sedimentarios. Esta inferencia concuerda con un origen deposicional distinto para cada uno de estos dos cuerpos sedimentarios.

\subsection{Fábricas arqueológicas}

Ningún nivel presenta una orientación preferencial con valores de $\mathrm{R} \%$ distantes de las fábricas lineares. Únicamente los datos de la unidad 497C se aproximan a
Verificando la integridad del registro arqueológico: análisis de fábricas en las unidades arqueológicas del paleolítico medio/superior de la Cova Gran (Santa Linya, Lleida)

las fábricas lineares, permaneciendo en el campo de las planares (tabla 2). Tridimensionalmente, el vector propio principal (V1) se orienta hacia el NE en las unidades 497C, S1B, y S1C, mientras que en $497 \mathrm{D}$ se orientan hacia el NW. Del mismo modo se observan bajos ángulos de buzamiento. Por su parte, los índices $\mathrm{K}$ y C sitúan estas unidades en el sector de las fábricas planares, aunque con un grado de isotropía mayor que el observado en las fábricas sedimentarias (tabla 2 y figura 6).

La coincidencia de las fábricas sedimentológicas y arqueológicas es un indicador de procesos de reorganización postdeposicional que afecta a un depósito arqueológico (Bertran et al., 1997; Bertran y Lenoble, 2002). Sin embargo, en la Cova Gran las características intrínsecas de las fábricas arqueológicas permiten diferenciarlas de las fábricas sedimentológicas, que muestran un menor grado de isotropía (Benito-Calvo

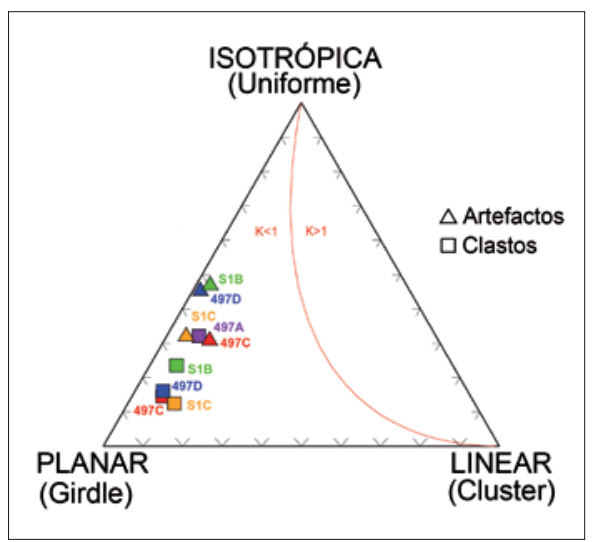

Figura 6. Diagrama ternario de Vollmer con la distribución de las fábricas asociadas a los clastos y los artefactos de los niveles arqueológicos discutidos en este trabajo. 
Miquel Roy Sunyer, Xavier Roda Gilabert, Alfonso Benito-Calvo, Jorge Martínez-Moreno, Rafael Mora Torcal

et al., 2009). Esta observación advierte que los materiales arqueológicos no han sido afectados por procesos sin/postdeposicionales severos que reorganicen la disposición originaria de los objetos. Una posible causa de estas divergencias, indicadas por una disposición aleatoria del material arqueológico, podría relacionarse con la actividad humana.

\section{Discusión: fábricas, procesos de formación y arqueoestratigrafía}

El análisis de las fábricas permite avanzar en la interpretación de procesos de formación del yacimiento de la Cova Gran. El depósito se sitúa en un abrigo de grandes dimensiones excavado en una pared de caliza muy diaclasada por la tectónica regional y afectada por procesos kársticos. La caída de fragmentos y bloques heterométricos genera contextos sedimentarios con texturas y morfologías que pueden ser caracterizadas mediante el análisis de fábricas. En consecuencia, este tipo de análisis permite evaluar en qué medida los procesos sin/postdeposicionales afectan a la distribución espacial y la organización de los materiales arqueológicos recuperados en los niveles de paleolítico medio y de paleolítico superior inicial de esta secuencia.

Los resultados obtenidos señalan que los clastos que integran los cuerpos sedimentarios tienden a orientaciones de tipo planar que se amoldan al relieve natural de la superficie del abrigo, mientras los artefactos presentan un patrón más isotrópico, no apreciándose orientaciones preferenciales. Esto implica que los mecanismos gravitacionales que originan la regularización de clastos y bloques no explican la disposición del material
Verificando la integridad del registro arqueológico: análisis de fábricas en las unidades arqueológicas del paleolítico medio/superior de la Cova Gran (Santa Linya, Lleida)

arqueológico sobre esas superficies. Al mismo tiempo denota que el material arqueológico no se reorganiza de forma significativa, ya sea por procesos tafonómicos o por fenómenos de escorrentía superficial (Benito-Calvo et al., 2009). Del mismo modo, las derivaciones obtenidas a partir de nuestras experimentaciones (Benito-Calvo et al., 2011) sugieren que la isotropía observada sobre el material arqueológico difiere de los patrones derivados de la acción de un pisoteo intensivo sobre materiales en superficie o semienterrados (figuras $3 \mathrm{c}$ y $3 \mathrm{~d}$ ).

Estas observaciones concuerdan con otros atributos como el buen estado de conservación de los artefactos, siendo escasos los restos líticos con alteraciones postdeposicionales como por ejemplo las piezas con retoques mecánicos o el redondeamiento de bordes y aristas, que denoten una intensa actividad hídrica. En este sentido, el análisis de las fábricas permite descartar que el torrente de Sant Miquel incida en la configuración de los cuerpos sedimentarios o sobre el material arqueológico preservado en el sector Rampa. Sin embargo, no puede descartarse su incidencia sobre el depósito, especialmente en el sector Plataforma. La oscilación altimétrica entre el sector Rampa (+ 9 m) y el sector Plataforma $(+3 \mathrm{~m})$ advierte que posiblemente el torrente incide sobre el abrigo, fosilizando la secuencia atribuida a la transición paleolítico medio/superior en esta zona concreta del yacimiento (figura 2). Este agente explicaría dos fenómenos. El primero es la inversión estratigráfica en la que los depósitos más antiguos se localizan en la zona más elevada del abrigo. El segundo, la interrupción abrupta de los niveles arqueológicos de paleolítico medio de esta 
Miquel Roy Sunyer, Xavier Roda Gilabert, Alfonso Benito-Calvo, Jorge Martínez-Moreno, Rafael Mora Torcal

secuencia, que no tienen continuidad en el sector Plataforma.

De igual forma, estos atributos sugieren que fenómenos mecánicos (como la incidencia del pisoteo) o ambientales (crioturbación) no han promovido una migración vertical generalizada de artefactos entre niveles. Señalamos que se han recuperado piezas fracturadas in situ que no han sido dispersadas, lo que denota una cierta integridad espacial incompatible con intensos procesos de reorganización postdeposicional.

Otra línea argumental que puede ayudar a precisar la resolución estratigráfica de un depósito es el análisis de las distribuciones en planta combinado con la proyección de secciones concretas del yacimiento. Este método constituye una técnica resolutiva a la hora de establecer relaciones entre materiales tanto en el plano horizontal como en el vertical y permite definir conjuntos de materiales espacial y temporalmente interrelacionados (Villa, 2004; Mora et al., 2008).

La geometría detectada para las unidades arqueológicas correspondientes al paleolítico medio y paleolítico superior inicial en el sector Rampa se articula a partir de la estratificación de niveles discretos y de poco espesor que se alternan con niveles estériles que caracterizan una secuencia de ocupaciones discontinuas (figura 7). Estos estériles sellan y preservan una sucesión de eventos ocupacionales diferentes que conforman unidades arqueológicas interestratificadas, que se identifican en planta y presentan un desarrollo vertical limitado (MartínezMoreno et al., 2010).

Esta observación conlleva varias implicaciones. Por un lado, se puede asumir que los niveles arqueológicos de la Cova Gran conforman superficies dinámicas resultado
Verificando la integridad del registro arqueológico: análisis de fábricas en las unidades arqueológicas del paleolítico medio/superior de la Cova Gran (Santa Linya, Lleida)

de un número indeterminado de ocupaciones del abrigo que se suceden a lo largo de una escala temporal difícil de precisar, pero que no puede considerarse muy prolongada (Martínez-Moreno et al., 2010; Mora et al., 2011). Asumimos que cada unidad arqueológica configura palimpsestos atribuibles a diferentes eventos de ocupación, pero que contiene una resolución arqueoestratigráfica que permite una identificación de conjuntos de materiales que se suceden dentro de un orden estratigráfico coherente. La extensión excavada, la dispersión vertical media, así como el número de artefactos líticos recuperados son factores indicativos de que nos encontramos ante conjuntos con los que abordar el análisis de las variaciones tecno-culturales que se detectan en esta secuencia (MartínezMoreno et al., 2010).

Este análisis evidencia que estos conjuntos no son el resultado de procesos de adición entre niveles atribuibles a periodos crono-culturales distintos, o el resultado de una recuperación inadecuada de la muestra arqueológica, factores reiteradamente aducidos al referir numerosas secuencias de Europa occidental que contienen niveles referidos con la «transición» paleolítico medio/superior (Zilhão, 2006; Zilhão y d'Errico, 1999).

Este escenario sugiere que los resultados derivados del estudio de los artefactos recuperados en las unidades arqueológicas referidas al fenómeno de cambio tecno-cultural detectado entre los niveles S1B/497D de la Cova Gran, puede aportar argumentos relevantes para analizar las causas relacionadas con la desaparición de los neandertales y la aparición de los primeros tecno-complejos de paleolítico superior en la Península Ibérica (Martínez-Moreno et al., 2010, 2012). Las implicaciones de esta 


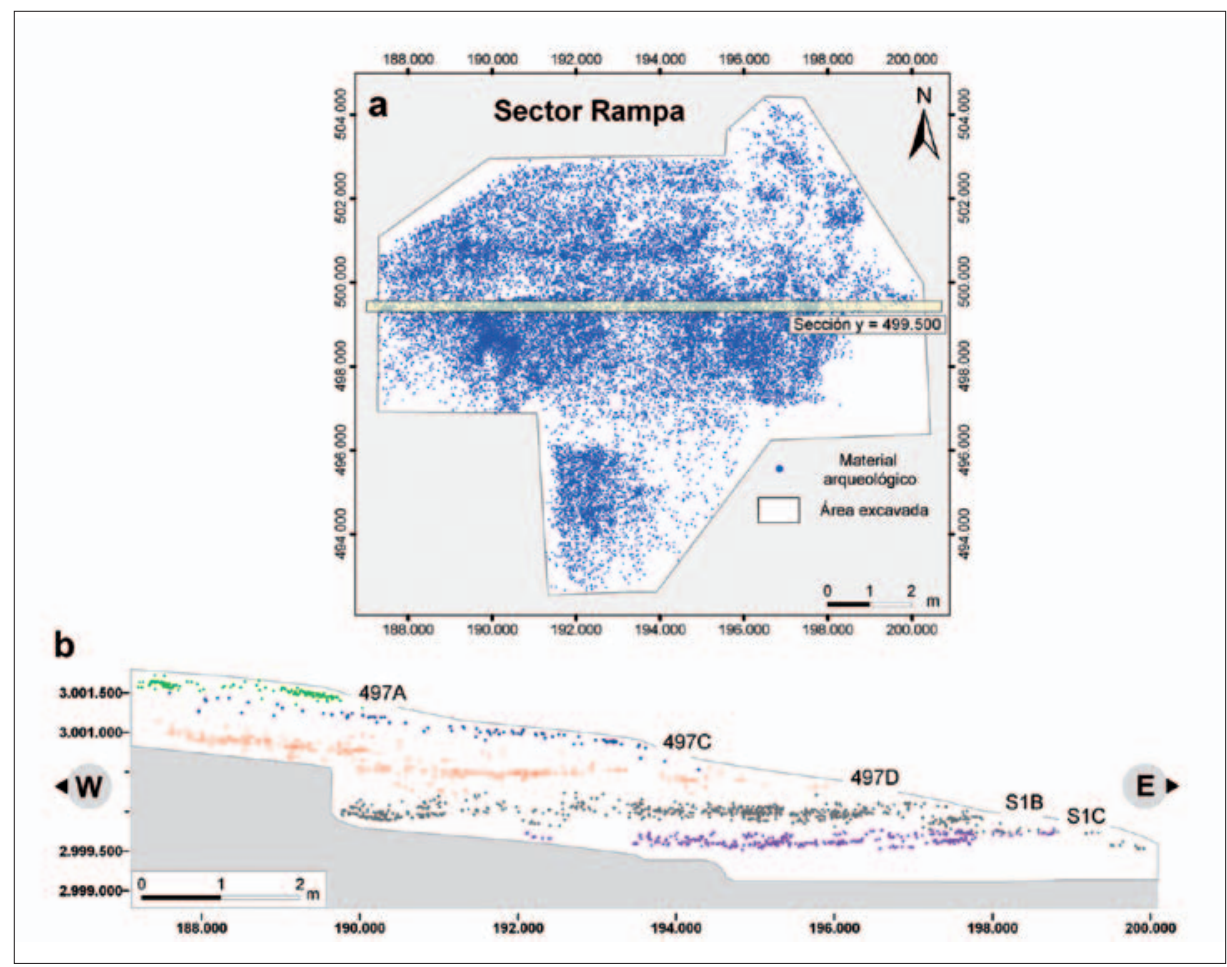

Figura 7. Arqueoestratigrafía del sector rampa de la Cova Gran. a) Distribución del material arqueológico correspondiente a la zona excavada y posición de la sección presentada; b) Sección $\mathrm{E}-\mathrm{W}$ en la que se observa la distribución vertical de los niveles arqueológicos de paleolítico medio (S1C y S1B) y paleolítico superior inicial (497A, 497C y 497D). La proyección de puntos permite identificar tramos estériles que separan las unidades arqueológicas a lo largo de su recorrido e indican una escasa migración vertical de los artefactos.

discusión, que merecerá ser examinada de forma detallada en futuras aportaciones, se sustentan en gran medida en los resultados que derivan del estudio de las fábricas presentados en este artículo.

\section{Conclusión}

La sistematización del análisis de fábricas que llevamos realizando en los últimos años nos ha permitido profundizar en aspectos críticos en el estudio de los conjuntos arqueológicos como son los procesos de formación y la integridad de estos. En este trabajo hemos presentado una síntesis metodológica que incluye los principales aspectos que conciernen el análisis de fábricas y la adaptación de las técnicas de análisis a nuestra metodología de excavación. La resolución de esta técnica para el caso concreto de la Cova 
Miquel Roy Sunyer, Xavier Roda Gilabert, Alfonso Benito-Calvo, Jorge Martínez-Moreno, Rafael Mora Torcal
Verificando la integridad del registro arqueológico: análisis de fábricas en las unidades arqueológicas del paleolítico medio/superior de la Cova Gran (Santa Linya, Lleida)
Gran nos ha sorprendido en gran medida por sus posibilidades en la evaluación de procesos sin/postdeposicionales, mostrando patrones de distribución de los elementos arqueológicos diferenciados de los procesos sedimentarios y de pisoteo. Las hipótesis con las que hemos trabajado en los últimos años son corrobo- radas por estos resultados, junto a otros caracteres de los conjuntos estudiados como son la distribución espacial de los restos o el estado de conservación. Estas nos hablan de un grado elevado de preservación de los palimpsestos del paleolítico medio final y paleolítico superior antiguo en la Cova Gran.

\section{Agradecimientos}

Esta investigación se inscribe dentro del proyecto Poblamiento y formas de vida durante el Pleistoceno Final y el Holoceno en el Prepirineo Oriental (HAR2010-15002E). Las excavaciones en el yacimiento de la Cova Gran son financiadas por el Servei d'Arqueologia i Paleontologia de la Generalitat de Catalunya y el Institut d'Estudis Ilerdencs-Diputació de Lleida. La Societat de Munts de Santa Linya autoriza la realización de trabajos de excavación en la Cova Gran. Esta es una contribución al grupo Cultura material y comportamiento humano de la Universitat Autònoma de Barcelona (2014SGR-84). Miquel Roy Sunyer (FI-DGR2012) y Xavier Roda Gilabert (FPIMICINN) son beneficiarios de becas de investigación. Rafael Mora agradece el apoyo del programa ICREA-Academia.

\section{Referencias bibliográficas}

BAILEY, G. 2007. "Time perspectives, palimpsests and the archaeology of time». Journal of Anthropological Archaeology 26: 198-223. $<$ http://dx.doi.org/10.1016/j.jaa.2006.08.002>

Benito-Calvo, A.; Martínez-Moreno, J.; Jordá Pardo, J.F.; De la Torre, I.; Mora TorCAL, R. 2009. "Sedimentological and archaeological fabrics in Palaeolithic levels of the South-Eastern Pyrenees: Cova Gran and Roca dels Bous sites (Lleida, Spain)». Journal of Archaeological Science 36: 2566-77. <http://dx.doi.org/10.1016/j.jas.2009.07.012>

Benito-Calvo, A.; Martínez-Moreno, J.; Mora, R.; Roy, M.; Roda, X. 2011. «Trampling experiments at Cova Gran de Santa Linya, Pre-Pyrenees, Spain: their relevance for archaeological fabrics of the Upper-Middle Paleolithic assemblages». Journal of Archaeological Science 38: 3652-61.

<http://dx.doi.org/10.1016/j.jas.2011.08.036> 
Miquel Roy Sunyer, Xavier Roda Gilabert, Alfonso Benito-Calvo, Jorge Martínez-Moreno, Rafael Mora Torcal
Verificando la integridad del registro arqueológico: análisis de fábricas en las unidades arqueológicas del paleolítico medio/superior de la Cova Gran (Santa Linya, Lleida)

Benito-Calvo, A.; De la Torre, I. 2011. "Analysis of orientation patterns in Olduvai Bed I assemblages using GIS techniques: implications for site formation processes». Journal of Human Evolution 62: 50-60. <http://dx.doi.org/10.1016/j.jhevol.2011.02.011>

BENN, D.I. 1994. "Fabric shape and the interpretation of sedimentary data». Journal of Sedimentary Research A64: 910-15.

Benn, D.I.; Ringrose, T.J. 2001. «Random variation of a fabric eigenvalues: implications for the use of A-Axis fabric data to differentiate till facies». Earth Surface Processes and Landforms 26: 295-306. <http://dx.doi.org/10.1002/1096-9837(200103)26:3<295::AID-ESP159>3.0.CO;2-1>

Bernaldo de Quirós, F.; Maillo, J.M.; Neira, A. 2008. "The place of unit 18 of Castillo cave in the Middle to Upper Paleolithic transition». Eurasian Prehistory 5: 57-72.

Bertran, P. 1994. «Dégradation des niveaux d'occupation paléolithiques en contexte périglaciaire: exemples et implications archéologiques». Paleo 6: 285-302.

Bertran, P.; Teixer, J-P. 1995. «Fabric Analysis: Application to Paleolithic sites». Journal of Archaeological Science 22: 521-35. <http://dx.doi.org/10.1006/jasc.1995.0050>

Bertran, P.; Hétu, B., Texier, J.-P.; Steijn, H. 1997. «Fabric characteristics of subaerial slope deposits». Sedimentology 44: 1-16. <http://dx.doi.org/10.1111/j.1365-3091.1997.tb00421.x>

Bertran, P.; Lenoble, A. 2002. «Fabriques des niveaux archéologiques: méthode et premier bilan des apports à l'étude taphonomique des sites paléolithiques». Paleo 14: 13-28.

Bar-Yosef, O.; Bordes, J.-G. 2010. "Who were the makers of the Châtelperronian culture?», Journal of Human Evolution 59: 586-93. <http://dx.doi.org/10.1016/j.jhevol.2010.06.009>

Bordes, J.-G. 2002. "Lithic taphonomy of the Châtelperronian/Aurignacian interstratifications in Roc de Combe and Le Piage (France)». En ZilHão, J.; D’ERrico, F. (eds): The chronology of the Aurignacian and of the transitional technocomplexes dating, stratigraphies, cultural implications, 223-44. Trabalhos de Arqueologia 33. Lisboa: Instituto Português de Arqueologia.

Butzer, K. 1982. Archaeology as Human Ecology. Cambridge: Cambridge University Press. <http://dx.doi.org/10.1017/CBO9780511558245>

Cahen, D.; Keeley, L.; Van-Noten, F. 1979. "Stone tools, toolkits, and human behavior in prehistory». Current Anthropology 20: 661-83. $<$ http://dx.doi.org/10.1086/202371>

Cziesla, E.; Eïckhoff, S.; Arts, N.; Winter, D. (Ed.) 1990. The Big Puzzle. International symposium on refitting stone artefacts. Studies in Modern Archaeology, 1.

Curray, J.R. 1956. "Analysis of two-dimensional orientation data». Journal of Geology, 64: $117-34$. <http://dx.doi.org/10.1086/626329>

Dibble, H. L.; Chase, P. G. ; McPherron S.; Tuffreau, A. 1997. «Testing the reality of a "living floor" with archaeological data». American Antiquity, 62: 629-51. <http://dx.doi.org/10.2307/281882>

Eren, M.I.A.; Durant, A.; Neudorf, C.; Haslam, M.; Shipton, C.; Bora, J.; Korissetar, R.; Petraglia, M. 2010. «Experimental examination of animal trampling effects on artefact movement in dry and water saturated substrates: a test case from South India». Journal of Archaeological Science 37: 3010-21. $<$ http://dx.doi.org/10.1016/j.jas.2010.06.024>

Gifford, D.; Behrensmeyer, A. 1977. «Observed formation and burial of a recent human occupation site in Kenya». Quaternary Research 8: 245-66.

<http://dx.doi.org/10.1016/0033-5894(77)90071-0> 
Miquel Roy Sunyer, Xavier Roda Gilabert, Alfonso Benito-Calvo, Jorge Martínez-Moreno, Rafael Mora Torcal
Verificando la integridad del registro arqueológico: análisis de fábricas en las unidades arqueológicas del paleolítico medio/superior de la Cova Gran (Santa Linya, Lleida)

Gifford-Gonzalez, D.; Damrosch, D.; Damrosch J.; Thunen, R. 1985. «The third dimension in site structure: an experiment in trampling and vertical dispersal». American Antiquity 50: 803-18. <http://dx.doi.org/10.2307/280169>

Gravina, B.; Mellars, P.; Ramsey, C. B. 2005. «Radiocarbon dating of interstratified Neanderthal and early modern human occupations at the Chatelperronian type-site». Nature, $438,51-56$. <http://dx.doi.org/10.1038/nature04006>

KJAER, K.H.; KRÜGER, J. 1998. «Does clast size influence fabric strength?», Journal of Sedimentary Research 68: 746-49. <http://dx.doi.org/10.2110/jsr.68.746>

Kroll, E.; Price. T. (Ed.) 1991. The interpretation of archaeological spatial patterning. Nueva York: Plenum Press.

Lenoble, A.; Ortega, I.; Bourguignon, L. 2000. «Processus de formation du site moustérien de Champs-de-Bossuet (Gironde)». Paleo 12: 413-25.

Lenoble, A.; Bertran, P. 2004. «Fabric of Palaeolithic levels: methods and implications for site formation processes». Journal of Archaeological Science, 31: 457-69. <http://dx.doi.org/10.1016/j.jas.2003.09.013>

Lenoble, A.; Bertran, P.; Lacrampe, F. 2008. «Solifluction-induced modifications of archaeological levels: simulation based on experimental data from a modern periglacial slope and application of French Palaeolithic sites». Journal of Archaeological Science, 35: 99-110. <http://dx.doi.org/10.1016/j.jas.2007.02.011>

Leroi-Gourhan, A.; Brézillon, M. 1966. "L’habitation magdalénienne $\mathrm{n}^{\circ} 1$ de Pincevent près Monterau (Seine-et-Marne)». Gallia préhistoire, 9: 263-385.

Malinsky-Buller, A.; Hovers, E.; Marder, O. 2011. "Making time: "Living floors", "palimpsests" and site formation processes - A perspective from the open-air Lower Paleolithic site of Revadim Quarry, Israel». Journal of Anthropological Archaeology, 30: 89-101. $<$ http://dx.doi.org/10.1016/j.jaa.2010.11.002>

Martínez-Moreno, J.; Mora, R.; De la Torre, I. 2010. «The Middle-to-Upper Palaeolithic transition in Cova Gran (Catalonia, Spain) and the extinction of Neanderthals in the Iberian Peninsula». Journal of Human Evolution, 58: 211-26. $<$ http://dx.doi.org/10.1016/j.jhevol.2009.09.002>

Martínez-Moreno, J.; González Marcén, P.; Mora Torcal, R. 2011. «Data matrix (DM) codes: A technological process for the management of the archaeological record». Journal of Cultural Heritage, 12(2): 134-39. <http://dx.doi.org/10.1016/j.culher.2010.10.001>

Martínez-Moreno, J.; Mora, R.; De la Torre Sáinz, I.; Benito, A. 2012. «The role of flakes in the early Upper Palaeolithic assemblage of Cova Gran de Santa Linya (Southeastern Prepyrenees, Spain)». En Pastoors A.; Peresani, M. (eds): Flakes not Blades: The role of flake production at the onset of the Upper Palaeolithic in Europe. Wissenschaftliche Schriften des Nanderthal Museums 5: 85-104. Mettmann.

McPherron, S.J.P. 2005. «Artefact orientation and site formation processes from total station proveniences». Journal of Archaeological Science, 32: 1003-14. <http://dx.doi.org/10.1016/j.jas.2005.01.015>

Mellars, P.; Gravina, B.; Ramsey, C. B. 2007. "Confirmation of Neanderthal/modern human interstratification at the Chatelperronian type-site». PNAS 104(9): 3657-62. <http://dx.doi.org/10.1073/pnas.0608053104>

Mills, H. H. 1983. "Clast-fabric strength in hillslope colluvium as a function of slope angle». Geografiska Annaler, 65 (3/4): 255-62. <http://dx.doi.org/10.2307/520590> 
Miquel Roy Sunyer, Xavier Roda Gilabert, Alfonso Benito-Calvo, Jorge Martínez-Moreno, Rafael Mora Torcal
Verificando la integridad del registro arqueológico: análisis de fábricas en las unidades arqueológicas del paleolítico medio/superior de la Cova Gran (Santa Linya, Lleida)

Mora, R. 1994. «El Sistema Lógico Analítico». En Merino, J.M. (Ed.), Tipología Lítica (pp. 368-86). Munibe 9. Donostia.

Mora, R.; Martínez-Moreno, J.; Casanova, J. 2008. "Abordando la noción de "variabilidad musteriense" en Roca dels Bous (Prepirineo suroriental, Lleida)». Trabajos de Prehistoria, 65(2): 13-28.

Mora, R.; Benito-Calvo, A.; Martínez-Moreno, J.; González Marcen, P.; De la Torre, I. 2011. "Chrono-stratigraphy of the Upper Pleistocene and Holocene archaeological sequence in Cova Gran (South-Eastern Pre-Pyrenees, Iberian Peninsula)». Journal of Quaternary Science 26: 635-44. $<$ http://dx.doi.org/10.1002/jqs.1486>

Nielsen, A. 1991. "Trampling the archaeological record: an experimental study». American Antiquity 56: 483-503. $<$ http://dx.doi.org/10.2307/280897>

PeÑa, J. L. 1983. La Conca de Tremp y sierras prepirenaicas comprendidas entre los ríos Segre y Noguera Ribagorzana. Estudio geomorfológico. Cartografía. Lleida, Instituto de Estudios Ilerdenses.

Pizarro, J; Roy, M.; Roda, X.; Vega, S.; Samper, S.; Plasencia, J.; Casanova, J.; LópezPrat, M.; González Marcén, P.; Martínez-Moreno, J.; Mora, R. 2013. "Nous elements de reflexió al voltant del poblament del Prepirineu Oriental al llarg del plistocè superior i l'holocè». III Col.loqui d'Arqueologia d'Odèn (el Solsonès). Darreres investigacions al Prepirineu Lleidatà 2009-2011. Odèn.

Pocoví, A. 1978. "Estudio geológico de las Sierras Marginales Catalanas». Acta Geológica Hispánica, 13 (3): 73-79.

Rico Herrero, M. 2004. Las paleocrecidas en la cuenca media del rio Segre durante el Pleistoceno superior-Holoceno: registros morfosedimentarios y análisis hidrológico. Tesis doctoral inédita. Universidad de Zaragoza.

Schiffer, M.B. 1983. "Toward the identification of formation processes». American Antiquity 48: 675-705. $<$ http://dx.doi.org/10.2307/279771>

- 1987. Formation Processes of the Archaeological Record. University of New Mexico Press, Albuquerque.

SтосктоN, E.D. 1973. «Shaw's Creek Shelter: human displacement of artefacts and its significance». Mankind 9: 112-17.

Texier, J.P. 2000. «A propos des processus de formation des sites préhistoriques». Paleo, 12: 379-86.

Theunissen, R.; Balme, J.; Beck, W. 1998. «Headroom and human trampling: cave ceiling height determines the spatial patterning of stone artefacts at Petzkes Cave, northern New South Wales». Antiquity, 72: 80-89.

Villa, P. 1982. «Conjoinable pieces and site formation processes». American Antiquity, 47: 276-290. <http://dx.doi.org/10.2307/279901>

- 2004. "Taphonomy and stratigraphy in European prehistory». Before Farming 1: 1-20.

Villa, P.; Courtin, J. 1983. «The interpretation of stratified sites: a view from underground». Journal of Archaeological Science, 10: 267-81. <http://dx.doi.org/10.1016/0305-4403(83)90011-0>

Vollmer, F.W. 1989. "A triangular fabric plot with applications for structural analysis». American Geophysical Union Transactions, 70: 463.

Watson, G. S. 1965. «Equatorial distribution on a sphere». Biometrika, 52 (1/2): 193-201. <http://dx.doi.org/10.2307/2333824>

- 1966. «The statistics of orientation data». Journal of Geology, 54: 786-97. 
Woodcock, N.H. 1977. «Specification of fabric shapes using an eigenvalue method». Geological Society of America Bulletin, 88: 1231-36. $<$ http://dx.doi.org/10.1130/0016-7606(1977)88<1231:SOFSUA>2.0.CO;2>

Woodcock, N.H.; NaYlor, M.A. 1983. "Randomness testing in three-dimensional orientation data». Journal of Structural Geology, 5: 539-48. <http://dx.doi.org/10.1016/0191-8141(83)90058-5>

Zilhão, J. 2006. "Chronostratigraphy of the Middle-to-Upper Paleolithic transition in the Iberian Peninsula». Pyrenae, 37: 7-84.

Zilhão, J.; D’Errico, F. 1999. "The chronology and taphonomy of the earliest Aurignacian and its implications for the understanding of Neandertal extinction». Journal of World Prehistory, 13: 1-68. <http://dx.doi.org/10.1023/A:1022348410845>

Zilhâo, J.; D’Errico, F.; Bordes, J-G.; Lenoble, A.; Texier J. P.; Rigaud, J. P. 2006. "Analysis of Aurignacian interstratification at the Châtelperronian-type site and implications for the behavioral modernity of Neandertals». PNAS, 103(33): 12643-48. <http://dx.doi.org/10.1073/pnas.0605128103>

- 2008. "Grotte des Fées (Châtelperron). History of research, stratigraphy, datting and archeology of the Châtelperronian type-site». Paleoanthropology, 2008: 1-42. 
